\title{
Development of a novel animal model to differentiate radiation necrosis from tumor recurrence
}

\author{
Sanath Kumar, \\ Department of Radiation Oncology, Henry Ford Health System, Detroit, MI 48202, USA
}

Ali S. Arbab,

Department of Radiology, Henry Ford Health System, Detroit, MI 48202, USA

Rajan Jain, Department of Radiology, Henry Ford Health System, Detroit, MI 48202, USA

Jinkoo Kim, Department of Radiation Oncology, Henry Ford Health System, Detroit, MI 48202, USA

Ana C. deCarvalho, Hermelin Brain Tumor Center, Henry Ford Health System, Detroit, MI 48202, USA

Adarsh Shankar, Department of Radiology, Henry Ford Health System, Detroit, MI 48202, USA

Tom Mikkelsen, and Hermelin Brain Tumor Center, Henry Ford Health System, Detroit, MI 48202, USA

\section{Stephen L. Brown}

Department of Radiation Oncology, Henry Ford Health System, Detroit, MI 48202, USA

Sanath Kumar: skumar4@hfhs.org

\section{Abstract}

Distinguishing tumor progression from radiation necrosis after treatment in patients with brain tumors presents a clinical dilemma. A well-characterized, orthotopic rodent model of radiationinduced brain necrosis including a tumor is not currently available The objective of the study was to create focal radiation necrosis in rat brain bearing human glioblastoma (GBM) using stereotactic radiosurgery and confirm it by immuno-histological analysis. Nude rats implanted with primary GBM cells were irradiated using a stereotactic setup $(n=3)$ or received no radiation $(\mathrm{n}=3)$. Ten weeks after the implantation, growth of the tumor was confirmed by magnetic resonance imaging (MRI). For each animal, MRI and contrast-enhanced CT images were obtained and fused using registration software. The tumor was identified and delineated using the fused CT/ MR images. A treatment plan was generated using a $4 \mathrm{~mm}$ radiosurgery cone such that one portion of the tumor receives $100 \%$ dose of $60 \mathrm{~Gy}$ sufficient to cause necrosis, whereas the tumor edge at depth receives only $50 \%$ or less dose, allowing for regrowth of the tumor. The brains were collected 10 weeks after irradiation and immuno-histological analysis was performed. Hematoxylin and eosin staining showed central liquefaction necrosis in the high dose region consistent with necrosis and viable tumor in the peripheral low dose region. Ki-67 staining showed highly proliferative tumor cells surrounding the necrotic parts of the tumor. Luxol fast blue and

(c) Springer Science+Business Media, LLC. 2012

Correspondence to: Sanath Kumar, skumar $4 @$ hfhs . org.

Conflict of Interest The authors declare that they have no conflict of interest. 
lectin staining showed demyelination and vascular injury in brain tissue consistent with radiation necrosis. We have developed a novel model of radiation necrosis in rats bearing glioma.

\section{Keywords}

Glioma; Radiation necrosis; Recurrence; Radiosurgery

\section{Introduction}

Malignant gliomas are one of the most aggressive tumors with a median survival of 12-15 months for patients with glioblastoma (GBM) and 2-5 years for patients with anaplastic gliomas [1]. The current standard of care involves surgery followed by radiation therapy and temozolomide [2]. Treatment response is typically evaluated with gadolinium-enhanced magnetic resonance imaging (MRI) using Macdonald criteria [3]. Unfortunately, even with multimodal treatment, the tumors can recur or progress during or after treatment requiring continuous imaging surveillance.

The enhancement seen with gadolinium MRI is secondary to breakdown of blood-brain barrier (BBB) and any process that disrupts BBB will in turn affect the area of enhancement, regardless of the underlying tumor activity. The post-treatment MRI scans from GBM patients often reveal non-specific enhancing lesions, many of which remain asymptomatic [4-7]. The increase in enhancement on the MRI could be due to treatment-induced BBB disruption, and radiation therapy is a well-documented cause of such enhancement [7, 8]. On the other hand, contrast enhancement on MRI could also be due to tumor recurrence.

Radiation therapy is known to cause two distinct changes in the brain parenchyma after treatment $[4,5]$. Pseudo-progression is seen as contrast enhancement on MRI usually within 3 months after treatment and exhibit spontaneous resolution. The incidence of pseudoprogression reported in the literature is $12-64 \%$ [9]. The less common but more serious change is the radiation necrosis seen 3-12 months after treatment, exhibiting characteristic histology and do not always show spontaneous resolution. The reported incidence of radiation necrosis ranges from 3 to $24 \%$, with a less than 5\% incidence after standard 60 Gy in $1.8-2$ Gy fraction $[10,11]$ and up to $20 \%$ with radiosurgery [12].

Several modalities, such as magnetic resonance spectroscopy, diffusion weighted MRI, MR and CT perfusion imaging and positron emission tomography have been used to differentiate tumor recurrence from treatment effects [13-16]. But these techniques lack specificity and need substantial improvement. Although gadolinium-enhanced MRI is a marker of BBB disruption, it does not reliably distinguish between tumor progression and radiation necrosis $[17,18]$. This distinction is clinically relevant as treatment-induced injury is treated conservatively, whereas tumor recurrence requires the use of additional treatment in the form of surgery, radiation and chemotherapy. Currently, the only reliable way to differentiate between recurrence and necrosis is surgical, which is associated with considerable morbidity. Even with surgery, choosing the area to target for tissue sampling can be difficult, and pathology results may be variable due to heterogeneous nature of gliomas. In order to elucidate the treatment response and molecular changes following different therapies, orthotopic GBM models are routinely used [19]. Investigators have also created radiation-induced injury models in animal brain [20-22], but a model mimicking radiation necrosis plus recurrent GBM is still lacking. Thus there is an urgent need to develop an animal model of radiation necrosis and recurrent GBM in order to understand the basic mechanisms of tumor progression following radiation therapy. Also, there is a need for improving and developing novel non-invasive techniques that can reliably distinguish 
between recurrent tumor and radiation necrosis, which can only happen with a better understanding of the differences between these two pathologies on a molecular level and with imaging biomarkers which have a specific physiologic basis. To our knowledge, there is no pre-clinical model of radiation necrosis in the setting of glioma reported in the literature. Thus, the aim of the present study was to develop a model of radiation necrosis using primary human GBM in rat brain using stereotactic radiosurgery and confirm it by immuno-histological analysis.

\section{Materials and methods}

All experiments performed in this study were approved by Institutional Animal Care and Use Committee.

\section{Cell line}

Primary GBM cells were obtained from explanted tumor samples from patients using an IRB-approved protocol [23]. Tumor HF2303 was dissociated, and the cells were grown as neurospheres in neurosphere medium: DMEM/F12 medium containing $2 \mathrm{mM}$ L-glutamine and supplemented with N-2 (Gibco Invitrogen Cell Culture, Grand Island, NY), 0.05\% BSA, $25 \mu \mathrm{g} / \mathrm{ml}$ gentamicin, 50 units $/ \mathrm{ml}$ penicillin G sodium, $50 \mu \mathrm{g} / \mathrm{ml}$ streptomycin sulfate, 20 ng/ml EGF and 20 ng/ml bFGF (PeproTech, Rocky Hills, NJ). After 1-3 weeks, multicellular floating neurospheres formed and were dissociated in $\mathrm{Mg}^{2+}-\mathrm{Ca}^{2+}$-free PBS then harvested and resuspended at a concentration of $8 \times 10^{7}$ cells $/ \mathrm{ml}$ of serum free media; 5 $\mu l$ of the cell suspension was implanted into each rat brain.

\section{Animals}

Athymic male rats $(n=6)$ weighing $150-200$ grams obtained from Charles River laboratory (Frederick, MD) were used in all experiments. After tumor implantation, three animals received radiation using stereotactic radio-surgery and three animals were used as negative control for necrosis secondary to hypoxia.

\section{Tumor implantation}

Animals were anesthetized with $100 \mathrm{mg} / \mathrm{kg}$ ketamine and $15 \mathrm{mg} / \mathrm{kg}$ xylazine i.p. The surgical zone was swabbed with betadine solution, the eyes coated with Lacri-lube and the animal was immobilized in a small animal stereotactic device (Kopf, Cayunga, CA). After draping, a $1-\mathrm{cm}$ incision was made $2 \mathrm{~mm}$ to the right of the midline and $1 \mathrm{~mm}$ retroorbitally. The skull was exposed with cotton-tip applicators and an HP-4 dental drill bit was used with a micromanipulator to drill a hole $2 \mathrm{~mm}$ to the right of the bregma, taking care not to penetrate the dura. A \#2701 $10 \mu$ l Hamilton syringe with a 26-gauge needle containing 4 $\times 10^{5}$ tumor cells in $5 \mu \mathrm{l}$ was lowered to a depth of $3.5 \mathrm{~mm}$, and then raised to a depth of 2.5 $\mathrm{mm}$. The GBM cells were injected slowly at a rate of $0.5 \mu 1 / 30 \mathrm{~s}$ until the entire volume was injected.

\section{Radiosurgery plan}

Growth of tumors was confirmed with MRI scan obtained 10 weeks after initial implantation (Fig. 1a). For each animal, a contrast-enhanced CT images of $1 \mathrm{~mm}$ slice thickness were obtained and transferred to Brain Lab iPlan treatment planning system (Brainlab, Feldkirchen, Germany) using DICOM transfer protocol. MR images were acquired on the same day, transferred to the planning workstation and registered with CT. The tumor was identified and delineated on the fused CT/MRI image dataset. A radiotherapy plan was generated using a $4 \mathrm{~mm}$ radio-surgery cone using a single beam such that one portion of the tumor received $100 \%$ of 60 Gy dose in single fraction, which is sufficient to cause necrosis, 
whereas the tumor edge at depth received only $50 \%$ or less dose, allowing for re-growth of the tumor (Fig. 2). A portion of normal brain adjacent to the tumor was also exposed to dose of $60 \mathrm{~Gy}$.

\section{Irradiation}

The planned doses were delivered in single fraction using a Novalis unit (Brainlab, Feldkirchen, Germany) with $6 \mathrm{MV}$ photon at the dose rate of $800 \mathrm{MU} / \mathrm{min}$. In addition, in order to verify the dosimetry fall off of the $4 \mathrm{~mm}$ cone, we exposed a GAFCHROMIC ${ }^{\mathrm{TM}}$ EBT2 film (International Specialty Products, Wayne, NJ). The film was placed at isocenter in a solid water phantom with $1 \mathrm{~cm}$ buildup layer, and irradiated with $400 \mathrm{MU} 6 \mathrm{MV}$ photon beam (Fig. 3a). After $24 \mathrm{~h}$ post-coloration wait time, it was scanned with Epson Expression 10000XL document flat-bed scanner (Seiko Epson Corp, Nagano, Japan) using software SilverFast Ai (LaserSoft Imaging AG, Germany). The red channel of the scanned image was subsequently analyzed.

\section{Immuno-histochemical analysis}

Animals were sacrificed 10 weeks after radiation using $150-200 \mathrm{mg} / \mathrm{kg}$ of pentobarbital (administered by intravenous or intra-peritoneal injection) and then perfused with $100 \mathrm{ml}$ of saline and $100 \mathrm{ml}$ of $3 \%$ paraformaldehyde for histological analysis. The whole brain was collected and fixed in $4 \%$ paraformaldehyde and $3 \%$ sucrose. The fixed brain was placed in a 200-400 g coronal rat brain matrix (Activational Systems Inc., Warren, MI) and cut into 2$\mathrm{mm}$ blocks. Blocks grossly containing tumor or radiation injury were processed and paraffin embedded. The embedded blocks were cut into serial $10 \mu \mathrm{m}$ sections for histology. Hematoxylin and eosin (H\&E) staining was used to confirm the presence of tumor or radiation necrosis. Immuno-histochemistry using Ki-67 antibody was used to assess the proliferation status of the tumor cells. Luxol fast blue was used to stain myelin fibers and FITC-tagged tomato lectin was used as a marker of vascular endothelium.

\section{Results}

\section{Radiosurgery dose distribution}

As shown in the Fig. 3b, there was a steep dose fall off with the use of stereotactic radiosurgery. The dose intensity dropped from 100 to $50 \%$ within $2 \mathrm{~mm}$ on either side of the central axis.

\section{Radiosurgery treatment plan}

As shown in the treatment plan (Fig. 2), the lateral portion of the tumor received more than $60 \mathrm{~Gy}$ where as the medial side of the tumor received less than 50\% dose. We expected, based on our previous experience [24], that 50-60 Gy would be sufficient to generate radiation necrosis, whereas $30 \mathrm{~Gy}$ at the far edge of the field is sub-curative and allows for eventual regrowth of the tumor mass. The dose-volume histogram (Fig. 4), confirms that only a portion of the tumor got full dose of $60 \mathrm{~Gy}$.

\section{Immunohistochemical analysis}

H\&E staining of sections from control tumor showed no regions of necrosis (Fig. 5a-c). Radiated tumors showed central area of liquefactive necrosis surrounded by viable tumor (Fig. 5d-f). Further, H\&E staining revealed extensive angiogenesis and telangiectasia in the necrotic regions of the brain (Fig. 6c-d). Ki-67 staining confirmed the presence of central area of necrosis surrounded by highly proliferative tumor cells (Fig. 7c-d). Quantitative analysis showed $80 \% \mathrm{Ki}-67$ positive cells in the tumor region receiving sub-curative radiotherapy compared to $30 \%$ in control, un-irradiated tumor regions (Fig. 7a-b). Luxol 
fast blue staining revealed demyelination in the brain tissue around the tumor on the radiated side (Fig. 8b-c) compared to intact myelin on the contralateral normal hemisphere (Fig. 8de). FITC-tagged tomato lectin staining at the corresponding sites showed dilated blood vessels indicating vascular injury (Fig. 9c-d). All the three radiated animals showed histological changes consistent with necrosis.

\section{Discussion}

In the present study, we have developed a reproducible and clinically relevant experimental model of radiation necrosis in rats bearing human glioma treated with stereotactic radiosurgery. To our knowledge, this animal model is unique in exhibiting a combination of radiation necrosis and viable tumor.

Radiation-induced necrosis is one of the dreaded side effects in radiation treatment of central nervous system tumors. The time course of radiation-induced changes has been described as acute (days to weeks after irradiation); sub acute or early delayed (2-6 months after the completion of radiation); and late effects (6 months to years after the completion of radiation) [25]. Clinical symptoms include worsening neurologic signs and symptoms, seizures, increased intracranial pressure and neurocognitive decline [26]. White matter changes are the most common finding on imaging studies [27, 28]. At the cellular level, radiation necrosis is thought to be secondary from damage to vascular and glial tissues (25, 29, and 30). Characteristic histopathologic findings include white matter necrosis, demyelination, and vascular damage with increased vascular permeability [29-32].

The differentiation of recurrent or progressive tumor from radiation necrosis after radiotherapy often presents a dilemma to the radiologist as both entities typically demonstrate contrast enhancement on imaging studies. The distinction is further complicated by the use chemotherapy or anti-angiogenic treatments in addition to radiation therapy. Currently, the distinction between the two entities is made on the basis of clinical symptoms, serial imaging over a prolonged follow-up time or surgical biopsy. Hence there is an urgent need to develop non-invasive techniques to help better differentiate between the two distinct clinical entities.

There have been reports of radiation-induced necrosis in normal brain [20,33-37] or tumor models $[21,22]$ in animals. But a clinically relevant animal model with combination of radiation necrosis with viable tumor is surprisingly not discussed in the literature. In the present study, we used stereotactic setup to obtain differential dose distribution so as to create necrosis in normal brain and part of the tumor corresponding to the high dose region, and viable tumor in the low dose region. Our model with radiation-induced necrosis and viable tumor (Fig. 6c-d) seems to be clinically more relevant as it mimics the actual clinical scenario where there is mixture of radiation necrosis with viable or recurrent tumor and hence, potentially could be more useful in evaluating techniques differentiating radiation necrosis from recurrent tumor.

We also used Ki-67 staining to determine the proliferative status of the residual tumor cells that after radiation. To our surprise, there were a higher percentage of proliferative cells in the tumor that received sub-lethal irradiation (Fig. 7c-d) compared to the naive tumors (Fig. $7 \mathrm{a}-\mathrm{b})$. We hypothesize this to be due to repopulation by tumor cells in the low dose region. Radiation causes mitotic cell death due to DNA damage. The radiosurgery technique used causes differential dose distribution across the tumor. The tumor cells in the high dose region undergo irreparable DNA damage, whereas the cells in the lower dose region are able to repair sub-lethal damage and can further undergo rapid cell division resulting in increased Ki-67 staining compared to un-irradiated tumor. 
Currently, we are using this model for blood volume and quantitative permeability estimates using dynamic contrast-enhanced CT and MRI to differentiate radiation necrosis from recurrent tumor.

\section{Conclusion}

We have developed a novel model of radiation necrosis in rats bearing glioma using stereotactic radiosurgery. This model could benefit the experimental study of new imaging and treatment strategies involving radiation necrosis and tumor recurrence.

\section{References}

1. Wen PY, Kesari S. Malignant gliomas in adults. N Engl J Med. 2008; 359:492-507. [PubMed: 18669428]

2. Stupp R, Mason WP, van den Bent MJ, et al. Radiotherapy plus concomitant and adjuvant temozolomide for glioblastoma. N Engl J Med. 2005; 352(10):987-996. [PubMed: 15758009]

3. Macdonald DR, Cascino TL, Schold SC Jr, et al. Response criteria for phase II studies of supratentorial malignant glioma. J Clin Oncol. 1990; 8:1277-1280. [PubMed: 2358840]

4. Brandsma D, van den Bent MJ. Pseudoprogression and pseudoresponse in the treatment of gliomas. Curr Opin Neurol. 2009; 22(6):633-638. [PubMed: 19770760]

5. Clarke JL, Chang S. Pseudoprogression and pseudoresponse: challenges in brain tumor imaging. Curr Neurol Neurosci Rep. 2009; 9(3):241-246. [PubMed: 19348713]

6. Chamberlain MC, Glantz MJ, Chalmers L, et al. Early necrosis following concurrent Temodar and radiotherapy in patients with glioblastoma. J Neurooncol. 2007; 82(1):81-83. [PubMed: 16944309]

7. de Wit MC, de Bruin HG, Eijkenboom W, et al. Immediate post-radiotherapy changes in malignant glioma can mimic tumor progression. Neurology. 2004; 63(3):535-537. [PubMed: 15304589]

8. Chaskis C, Neyns B, Michotte A, et al. Pseudoprogression after radiotherapy with concurrent temozolomide for high-grade glioma: clinical observations and working recommendations. Surg Neurol. 2009; 72(4):423-428. [PubMed: 19150114]

9. Sanghera P, Perry J, Sahgal A, et al. Pseudoprogression following chemoradiotherapy for glioblastoma multiforme. Can J Neurol Sci. 2010; 37(1):36-42. [PubMed: 20169771]

10. Ruben JD, Dally M, Bailey M, et al. Cerebral radiation necrosis: incidence, outcomes, and risk factors with emphasis on radiation parameters and chemotherapy. Int J Radiat Oncol Biol Phys. 2006; 65:499-508. [PubMed: 16517093]

11. Emami B, Lyman J, Brown A, et al. Tolerance of normal tissue to therapeutic irradiation. Int J Radiat Oncol Biol Phys. 1991; 21:109-122. [PubMed: 2032882]

12. Marks LB, Yorke ED, Jackson A, et al. Use of normal tissue complication probability models in the clinic. Int J Radiat Oncol Biol Phys. 2010; 76:S10-S19. [PubMed: 20171502]

13. Jain R, Scarpace L, Ellika S, et al. First pass perfusion CT: initial experience in differentiating recurrent brain tumors from radiation effects/radiation necrosis. Neurosurgery. 2007; 61(4):778787. [PubMed: 17986939]

14. Hein PA, Eskey CJ, Dunn JF, et al. Diffusion-weighted imaging in the follow-up of treated highgrade gliomas: tumor recurrence versus radiation injury. Am J Neuroradiol. 2004; 25:201-209. [PubMed: 14970018]

15. Rachinger W, Goetz C, Pöpperl G, Gildehaus FJ, et al. Positron emission tomography with O-(2$[18 \mathrm{~F}]$ fluoroethyl)-L-tyrosine versus magnetic resonance imaging in the diagnosis of recurrent gliomas. Neurosurgery. 2005; 57(3):505-511. [PubMed: 16145529]

16. Tsuyuguchi N, Takami T, Sunada I, et al. Methionine positron emission tomography for differentiation of recurrent brain tumor and radiation necrosis after stereotactic radiosurgery-in malignant glioma. Ann Nucl Med. 2004; 18(4):291-296. [PubMed: 15359921]

17. Kumar AJ, Leeds NE, Fuller GN, et al. Malignant gliomas: MR imaging spectrum of radiation therapy- and chemotherapy-induced necrosis of the brain after treatment. Radiology. 2000; 217:377-384. [PubMed: 11058631] 
18. Mullins ME, Barest GD, Schaefer PW, et al. Radiation necrosis versus glioma recurrence: conventional MR imaging clues to diagnosis. Am J Neuroradiol. 2005; 26:1967-1972. [PubMed: 16155144]

19. Barth RF. Rat brain tumor models in experimental neuro-oncology: the 9L, C6, T9, F98, RG2 (D74), RT-2 and CNS-1 gliomas. J Neurooncol. 1998; 36(1):91-102. [PubMed: 9525831]

20. Zhou J, Tryggestad E, Wen Z, et al. Differentiation between glioma and radiation necrosis using molecular magnetic resonance imaging of endogenous proteins and peptides. Nat Med. 2011; 17(1):130-134. [PubMed: 21170048]

21. Kondziolka D, Lunsford LD, Claassen D, et al. Radiobiology of radiosurgery: Part II. The rat C6 glioma model. Neurosurgery. 1992; 31(2):280-287. [PubMed: 1325039]

22. Jost SC, Hope A, Kiehl E, et al. A novel murine model for localized radiation necrosis and its characterization using advanced magnetic resonance imaging. Int J Radiat Oncol Biol Phys. 2009; 75(2):527-533. [PubMed: 19735877]

23. deCarvalho AC, Nelson K, Lemke N, et al. Gliosarcoma stem cells undergo glial and mesenchymal differentiation in vivo. Stem Cells. 2010; 28(2):181-190. [PubMed: 19937755]

24. Arbab AS, Janic B, Jafari-Khouzani K, et al. Differentiation of glioma and radiation injury in rats using in vitro produce magnetically labeled cytotoxic T-cells and MRI. PLoS One. 2010; 5(2):e9365. [PubMed: 20195476]

25. Schultheiss TE, Kun LE, Ang KK, et al. Radiation response of the central nervous system. Int J Radiat Oncol Biol Phys. 1995; 31:1093-1112. [PubMed: 7677836]

26. Postma TJ, Klein M, Verstappen CC, et al. Radiotherapy-induced cerebral abnormalities in patients with low-grade glioma. Neurology. 2002; 59:121-123. [PubMed: 12105319]

27. Constine LS, Konski A, Ekholm S, et al. Adverse effects of brain irradiation correlated with MR and CT imaging. Int J Radiat Oncol Biol Phys. 1998; 15:319-330. [PubMed: 2841263]

28. Tsuruda JS, Kortman KE, Bradley WG, et al. Radiation effects on cerebral white matter: MR evaluation. Am J Roentgenol. 1987; 49:165-171. [PubMed: 3495977]

29. Wong CS, Van der Kogel AJ. Mechanisms of radiation injury to the central nervous system: implications for neuroprotection. Mol Interv. 2004; 4:273-284. [PubMed: 15471910]

30. Belka C, Budach W, Kortmann RD, et al. Radiation induced CNS toxicity: molecular and cellular mechanisms. Br J Cancer. 2001; 85:1233-1239. [PubMed: 11720454]

31. Fike JR, Sheline GE, Cann CE, et al. Radiation necrosis. Prog Exp Tumor Res. 1984; 28:136-151. [PubMed: 6484197]

32. Tofilon PJ, Fike JR. The radioresponse of the central nervous system: a dynamic process. Radiat Res. 2000; 153:357-370. [PubMed: 10798963]

33. Yamaguchi N, Yamashima T, Yamashita J. A histological and flow cytometric study of dog brain endothelial cell injuries in delayed radiation necrosis. J Neurosurg. 1991; 74(4):625-632. [PubMed: 2002376]

34. Lunsford LD, Altschuler EM, Flickinger JC, et al. In vivo biological effects of stereotactic radiosurgery: a primate model. Neurosurgery. 1990; 27(3):373-382. [PubMed: 1700326]

35. Kondziolka D, Lunsford LD, Claassen D, et al. Radiobiology of radiosurgery: Part I. The normal rat brain model. Neurosurgery. 1992; 31(2):271-279. [PubMed: 1513433]

36. Spiegelmann R, Friedman WA, Bova FJ, et al. LINAC radiosurgery: an animal model. J Neurosurg. 1993; 78(4):638-644. [PubMed: 8450338]

37. Tiller-Borcich JK, Fike JR, et al. Pathology of delayed radiation brain damage: an experimental canine model. Radiat Res. 1987; 110(2):161-172. [PubMed: 3575649] 


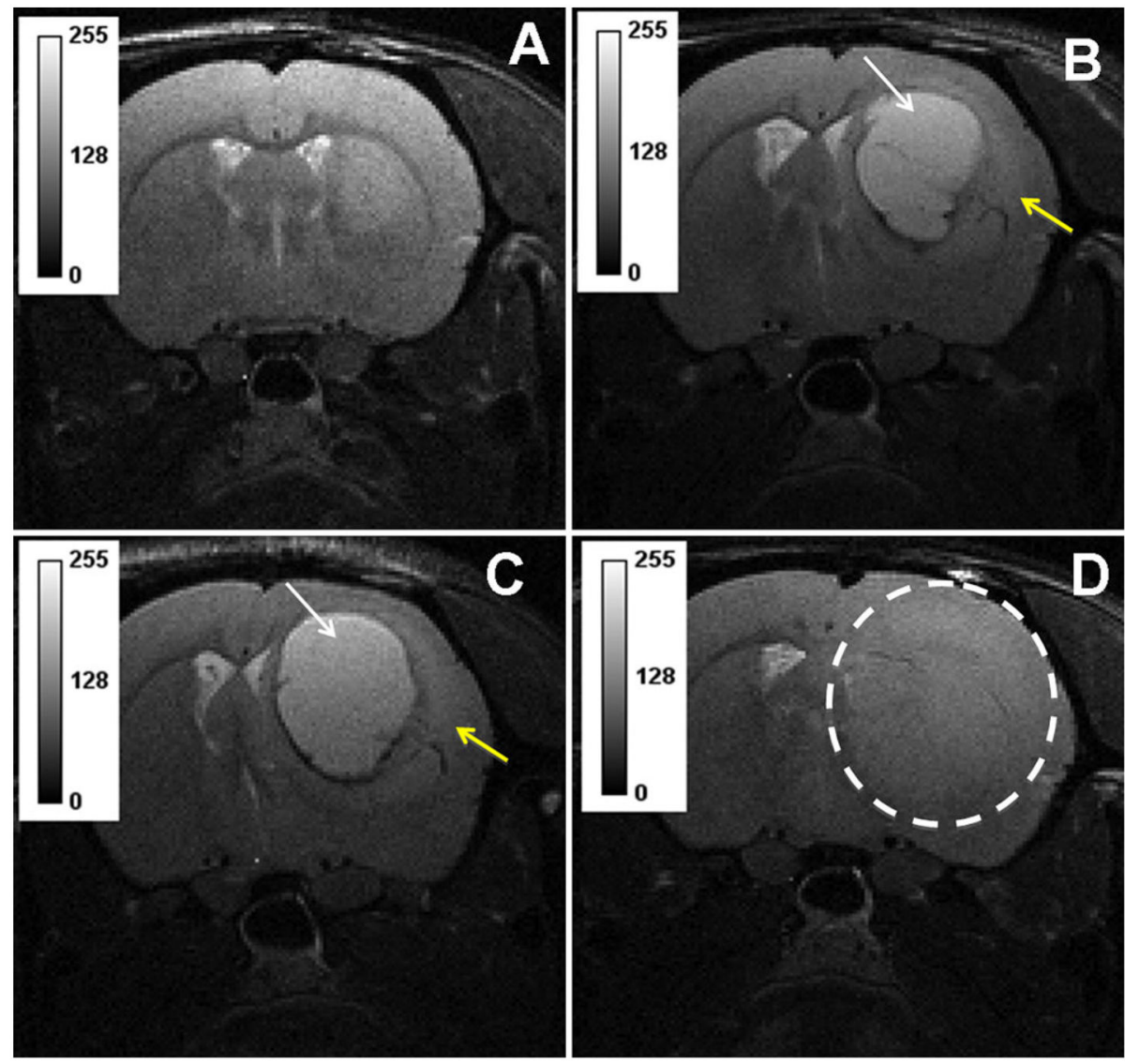

Fig. 1.

T2 weighted images from representative animal showing well established tumor 10 weeks after implantation (a). T2-hyperintense signal suggestive of radiation necrosis (white arrow) surrounded with infiltrating tumor (yellow arrow) was seen 7-8 weeks after stereo-tactic irradiation with single fraction dose of $60 \mathrm{~Gy}(\mathbf{b}-\mathbf{c})$. Control animal showed infiltrating tumor without any evidence of necrosis $(\mathbf{d})$ 


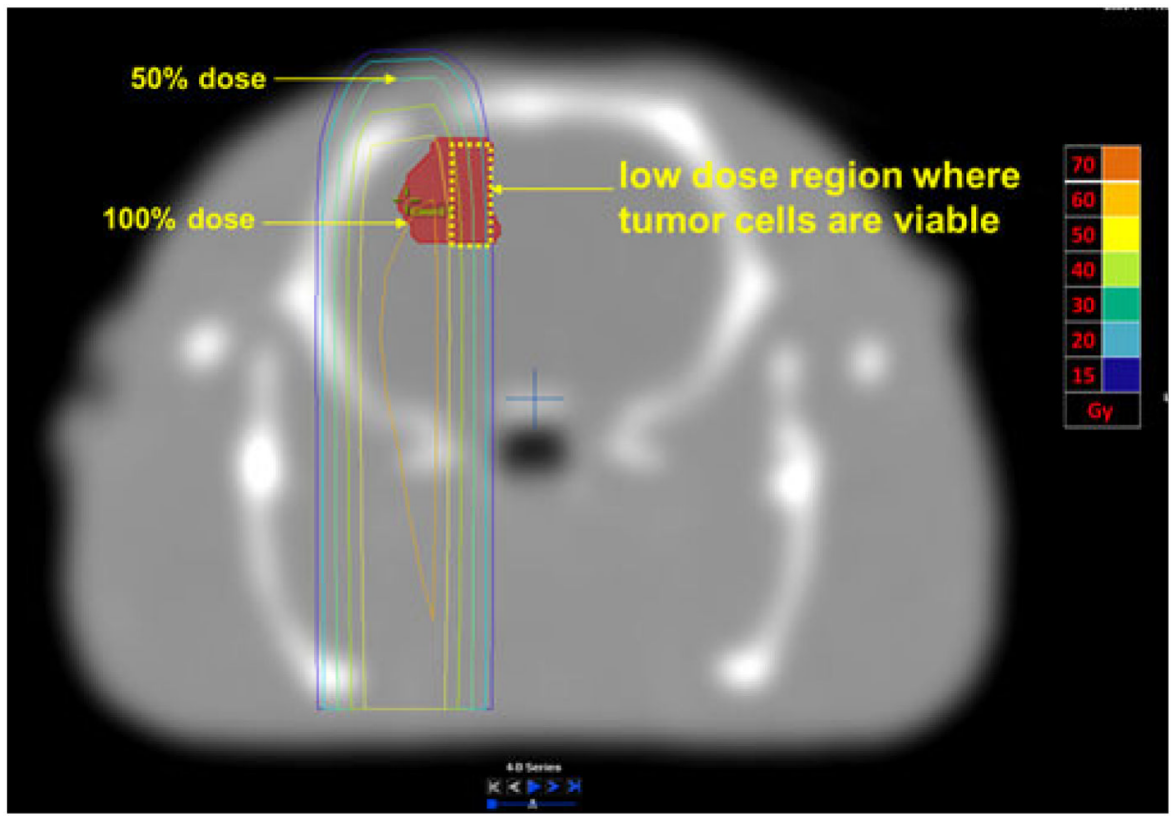

Fig. 2.

Proof of concept radiosurgery plan showing differential dose distribution. Only a portion of the tumor got $100 \%$ dose, while the tumor on the medial side received less than $50 \%$ dose. Tumor cells would be viable in the lower dose area allowing for regrowth of the tumor. Absolute doses are shown in the side bar 

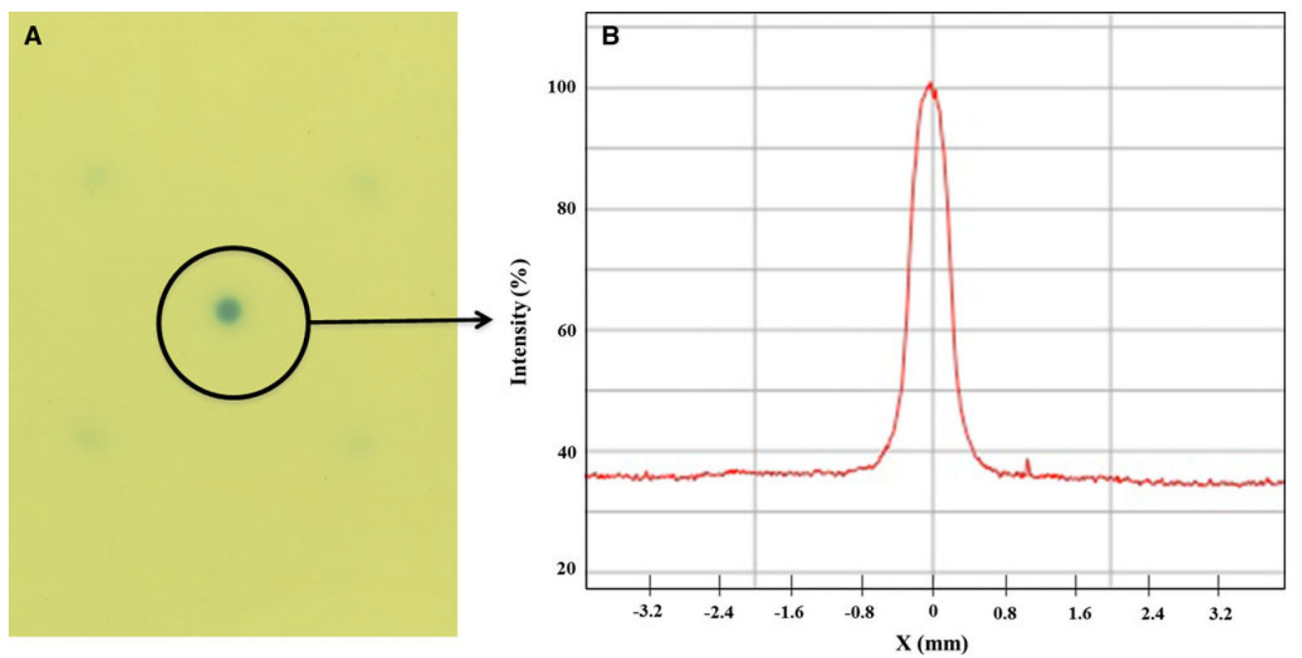

Fig. 3.

Dose falloff using a $4 \mathrm{~mm}$ cone; a a GAFCHROMIC film irradiated using 6MV radiosurgery beam and $\mathbf{b}$ the profile across the center showing sharp dose fall off from 100 to $50 \%$ dose 


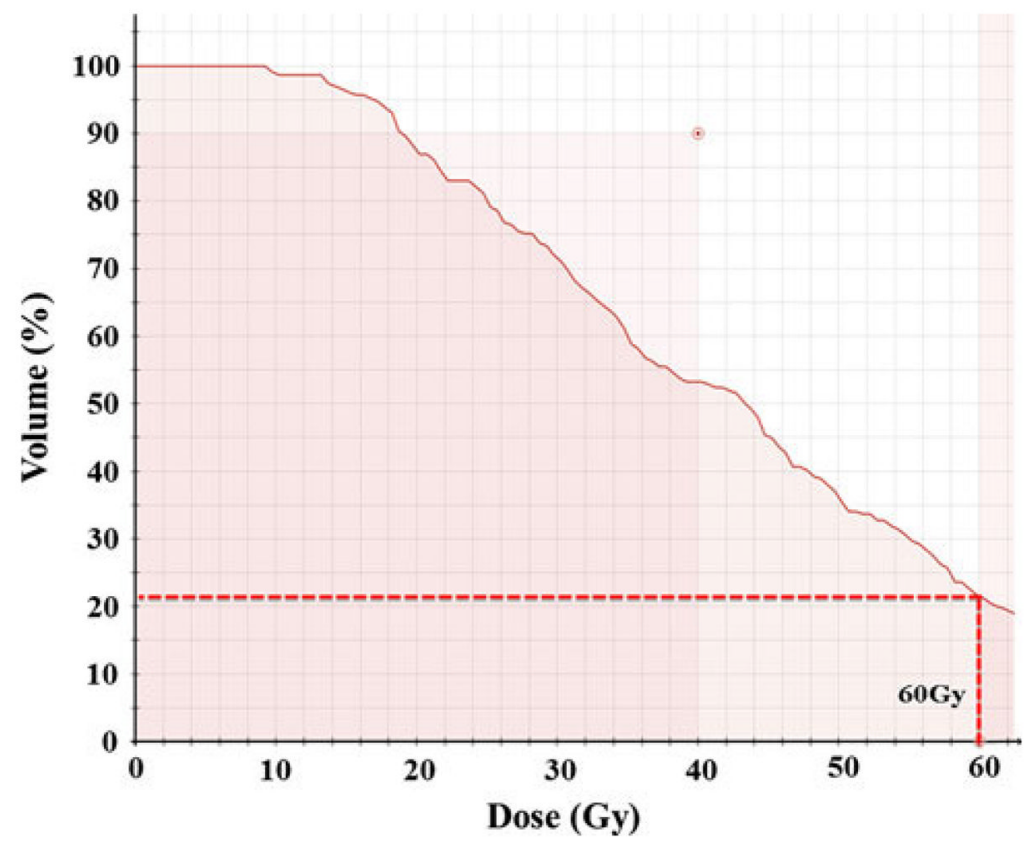

Fig. 4.

Dose-volume histogram showing only a portion of the tumor received $60 \mathrm{~Gy}$ enough to cause necrosis, while the tumor cells receiving sub-lethal dose would be viable 


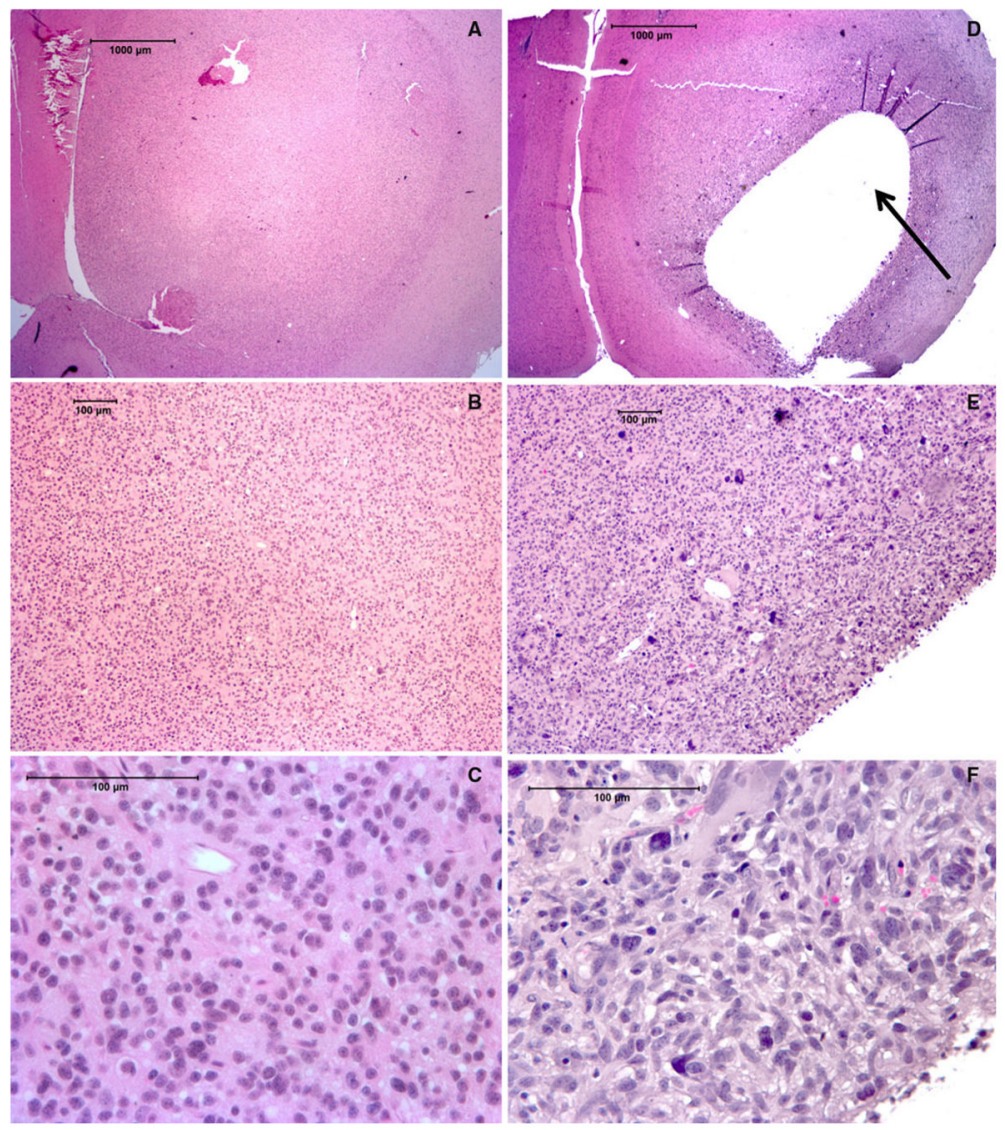

Fig. 5.

Histological H\&E staining showing control tumor with no necrosis at $2 \times, 10 \times$ and $40 \times$ magnifications (a-c respectively). Tumor receiving radiotherapy shows central liquefactive necrosis $($ arrow) $(\mathbf{d})$. Higher magnification views show necrotic region surrounded by viable tumor (e-f) 


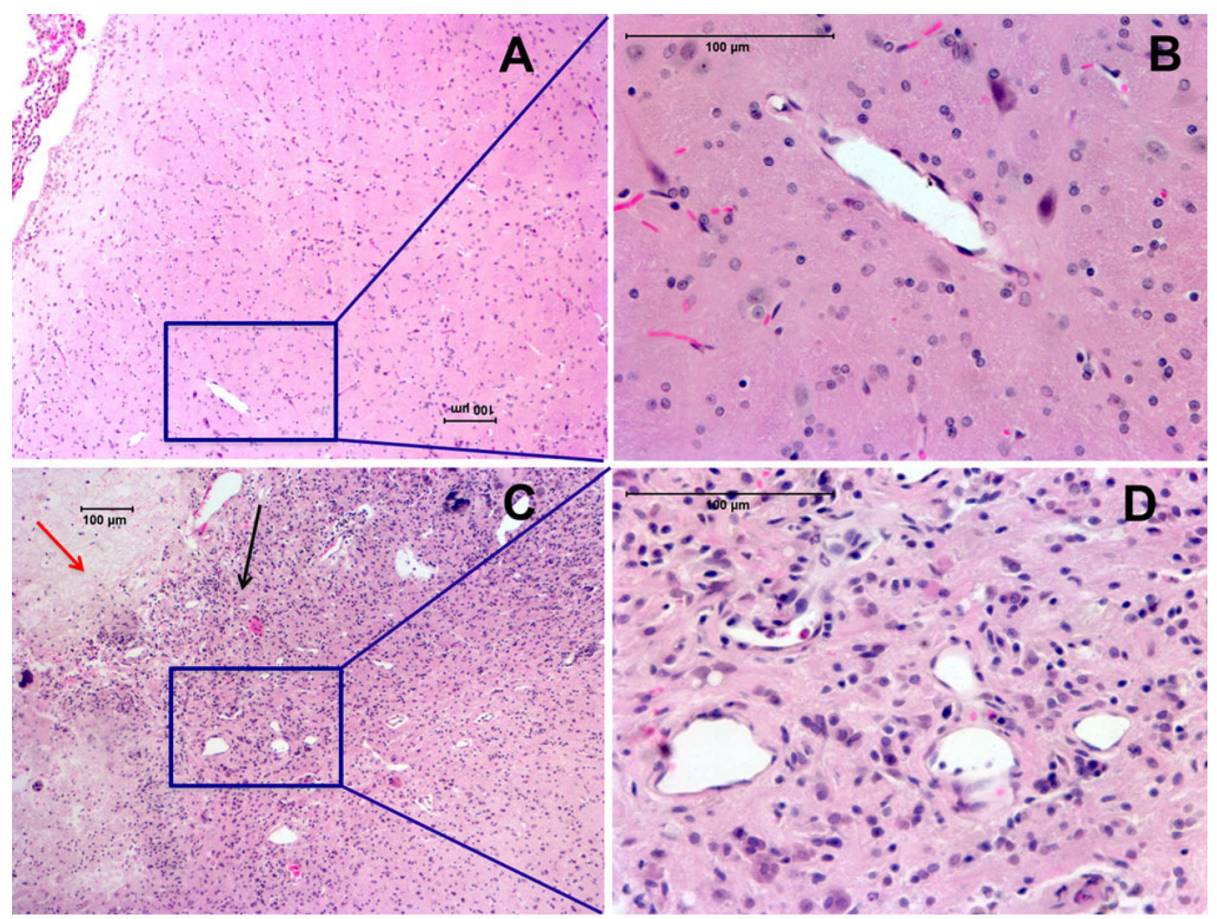

Fig. 6.

H\&E staining showing radiation-induced tumor necrosis (red arrow) along with viable tumor (black arrow) surrounded by necrosis in brain (c). Higher magnification of the necrotic region in brain revealed extensive angiogenesis and telangiectasia $(\mathbf{d})$. Contralateral side without necrosis showed normal looking blood vessels (a-b) 


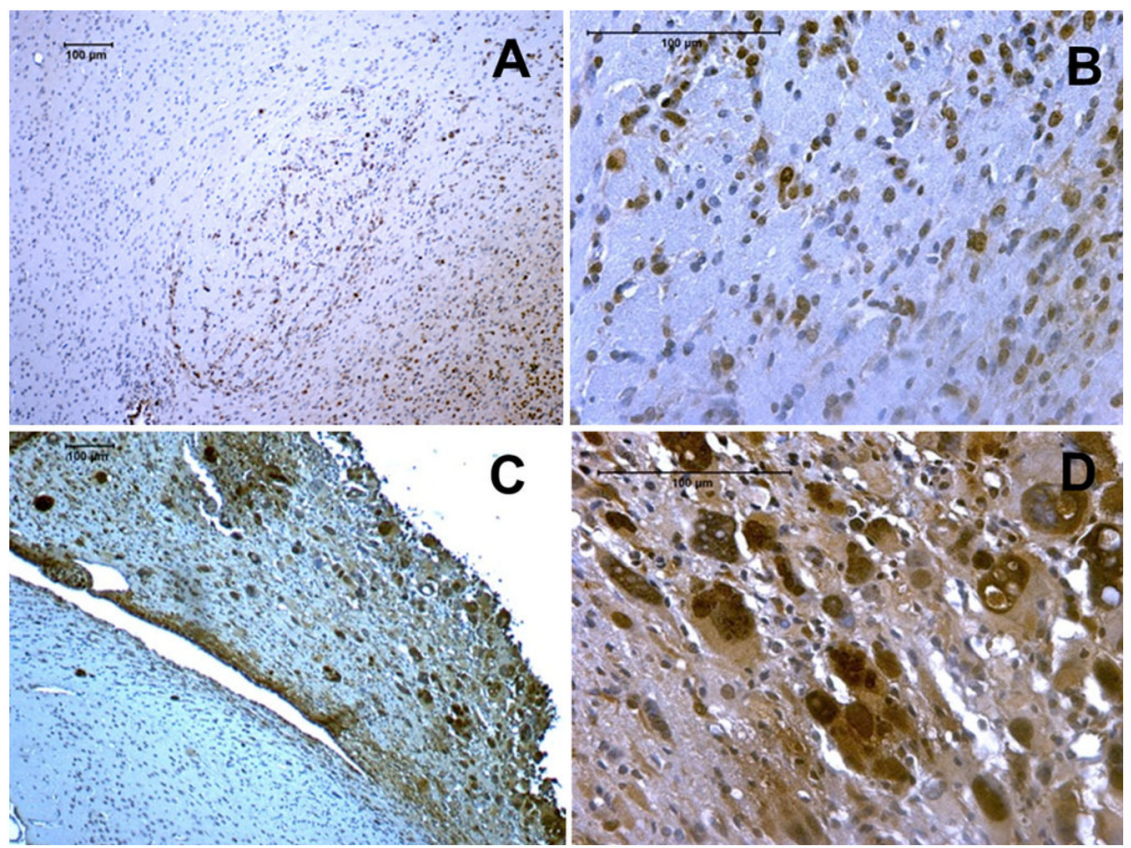

Fig. 7.

Ki-67 staining of control tumor (a-b) and after irradiation (c-d). Quantitative analysis showed increase in the percentage of Ki-67 positive cells in the tumor receiving sub-curative radiotherapy compared to control, un-irradiated tumors 


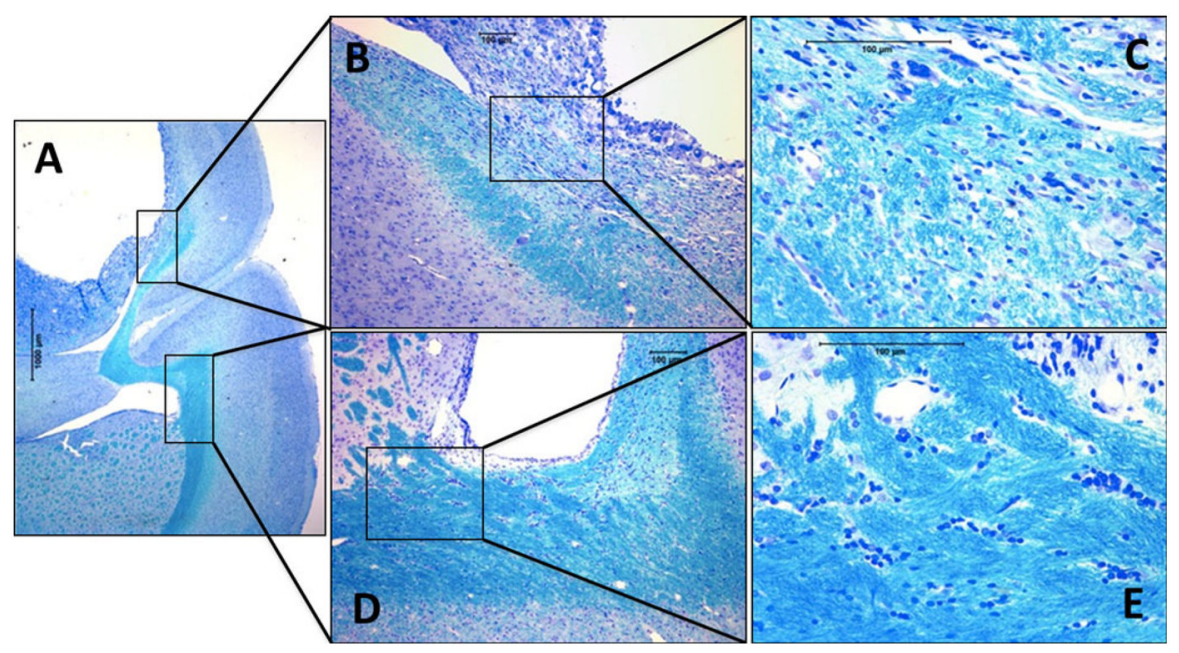

Fig. 8.

Luxol fast blue staining showing necrosis in the brain on the treated site compared to normal contralateral hemisphere (a). Higher magnification view revealed extensive loss of myelination in the brain tissue around the tumor site $(\mathbf{b}-\mathbf{c})$. Contralateral hemisphere showed normal brain tissue with intact myelin around the ventricle $(\mathbf{d}-\mathbf{e})$ 

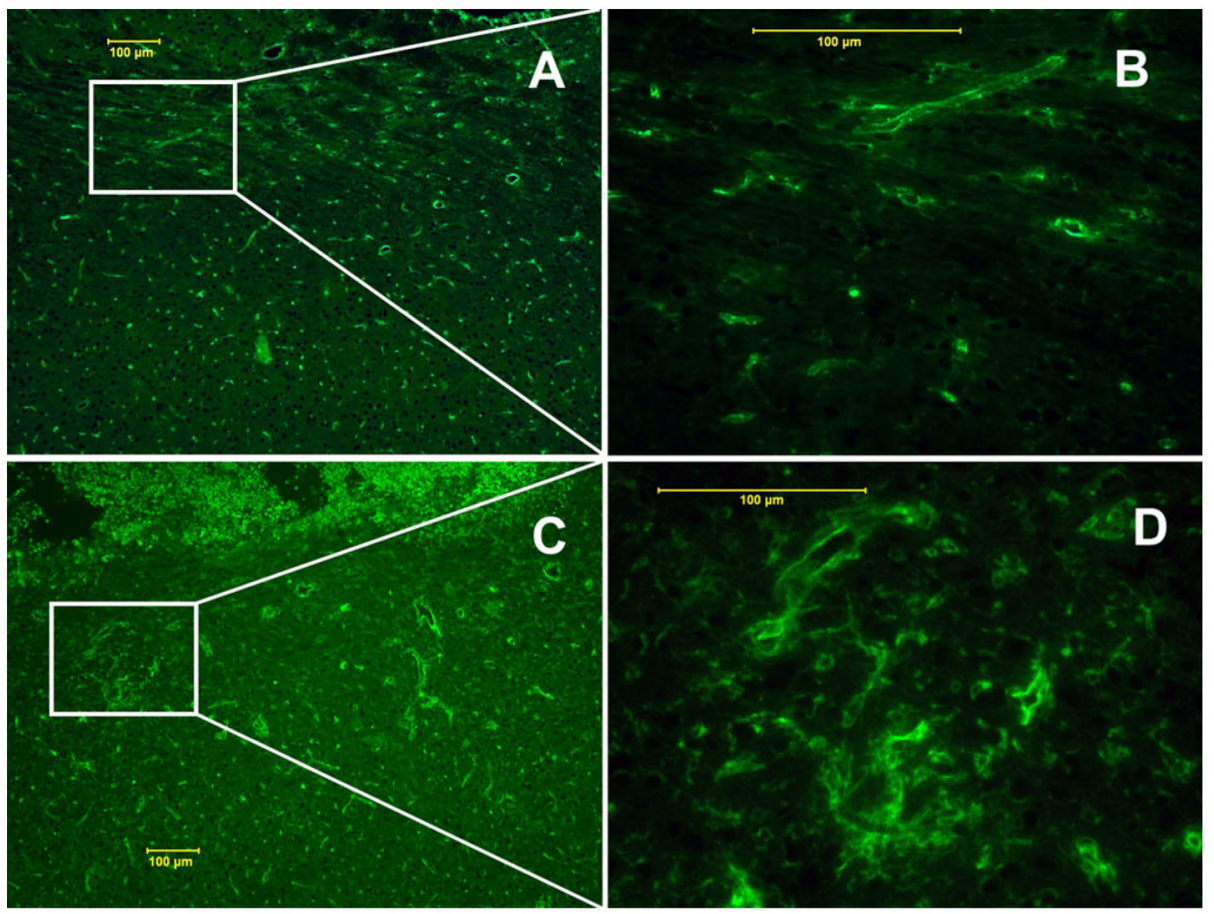

Fig. 9.

FITC-tagged tomato lectin staining showed loss of normal blood vessel morphology with evidence of dilatation at the site of radiation injury corresponding to demyelinated area (cd), in contrast to the normal vessel distribution seen in the contralateral hemisphere (a-b) 